\title{
Complex Dynamic Systems View on Conceptual Change: How a Picture of Students' Intuitive Conceptions Accrue From Dynamically Robust Task Dependent Learning Outcomes
}

\author{
I. T. KOPONEN, T. KOKKONEN AND M. NOUSIAINEN \\ Department of Physics, University of Helsinki (Finland)
}

\begin{abstract}
We discuss here conceptual change and the formation of robust learning outcomes from the viewpoint of complex dynamic systems (CDS). The CDS view considers students' conceptions as context dependent and multifaceted structures which depend on the context of their application. In the CDS view the conceptual patterns (i.e. intuitive conceptions here) may be robust in a certain situation but are not formed, at least not as robust ones, in another situation. The stability is then thought to arise dynamically in a variety of ways and not so much to mirror rigid ontological categories or static intuitive conceptions. We use computational modelling to understand the generic dynamic and emergent features of that phenomenon. The model is highly simplified and idealized, but it shows how context dependence, described here by an epistemic landscape structure, leads to the formation of context dependent robust states that can be viewed as attractors in learning, and how owing to the sharply defined nature of these states, learning appears as a progression of switches from one state to another, giving thus the appearance of conceptual change as switches from one robust state to another. Finally, we discuss the implications of the results in directing attention to the design of learning tasks and their structure, and how empirically accessible learning outcomes might be related to these underlying factors.
\end{abstract}

\section{Introduction}

The acquisition of scientific knowledge where learner's initial and personal concepts and conceptions gradually change towards scientific concepts is often referred to as conceptual change, with the aim to highlight the clear transition or even revolutionary-like transformation of learners' knowledge during that learning process (Chi 2005; Chi and Slotta 1993; diSessa 1993; diSessa and Sherin 1998; Reiner et al. 2000; Vosniadou \& Skopeliti 2014). Conceptual change has been described from many diverse viewpoints. At one end lies the "knowledge-as-theory" view, which claims that students' intuitive knowledge is theory-like and "coherent" (Vosniadou \& Skopeliti 2014) and at 
the other end of the continuum is the "knowledge-as-pieces" view, which views novices' knowledge as being fragmented, consisting of loosely connected pieces of knowledge (diSessa 1993; diSessa and Sherin 1998). Disagreement between these views of conceptual change centre on the basic elements of intuitive knowledge, their size and mutual relationships, as well as the nature of the change between the elements and their relations (Ozdemir \& Clark 2007). Numerous empirical studies of conceptual change have attempted to assess these questions but the results seem to depend so heavily on the theoretical background adopted for their interpretation that no basic agreement has emerged (diSessa et al. 2004; Russ et al. 2012; Wong 2016).

The recently suggested dynamic perspective (Brown 2014; Gupta et al. 2010; Hammer et al. 2005; Koponen et al. 2014, 2016) on students' intuitive conceptions and conceptual change attempts to provide a new picture based on complex dynamical systems thinking. This picture emphasises the nature of students' conceptions as multifaceted structures which depend on the context of their application. In the dynamic view the conceptual patterns (i.e. intuitive conceptions here) may be robust in a certain situation but are not formed, at least not as robust ones, in another situation. The stability is then thought to arise dynamically in a variety of ways and not so much to mirror rigid preconceptions or static intuitive conceptions (Hammer et al 2011, 2005; Gupta et al 2010).

A possibility that should be taken seriously is that what we think as intuitive conceptions, providing the furniture of students' thinking and reasoning, are after all so strongly dependent on context, instructional settings and individual learning histories that such conceptions should be approached as emergent cognitive epiphenomena, which are situational and which partially mirror the targeted scientific models that form the basis for the design of the instructional settings. We refer to such epiphenomenal conceptual structures simply as students' explanatory models.

In this study we discuss how the systemic view informed by the complex systems, views conceptual change as accruing from emergent robust learning outcomes. As a concrete example of learning we consider the extensively studied case of direct current (DC) electrical circuits (Koumaras et al. 1997; Reiner et al 2000; Chi 2005; Gupta et al. 2011; Koponen et al. 2014). In this case the target knowledge and learning situation can be modelled as learning a tiered system of models, where students are expected to learn a simple set of concepts, then relational schemes between the concepts and then apply them in simple concrete situations to give explanations. The picture provided here is highly simplified and idealized, but it shows how students' learning dynamics are affected by the instructional setting and how this leads to the formation of context dependent robust states, which are kinds of attractors in learning. Due to sharply defined nature of these states, dynamically continuous learning appears as a progression of discontinuous switches from one state to another, giving the appearance of conceptual change as a switch from one preexisting state to another. Finally, we discuss the implications of this new way to conceptualise conceptual change research.

\section{Learning Task and Students' Use of Explanatory Models}

The empirical research of learning DC-circuits has revealed that the students tend to use very similar types of explanatory models. Some researchers of conceptual change attribute these models to pre-existing ontological commitments (Reiner et al. 2000; Lee \& Law 2001), while some others see them as being more context dependent and possibly even artefacts of the empirical research setting (Gupta 2010). Nevertheless, most empirical studies, have revealed very similar collections of explanatory models although there are differences in details (McDermott \& Shaffer 1992; Koumaras et al. 1997; Engelhardt and Beichner 2004). The empirical data used here as a starting point consist of three different contexts I-III: 
I: Light bulbs in series. Two variants (a single light bulb and two light bulbs) in terms of the brightness of the bulbs are compared. This comparison produces evidence e 1 and e2.

II: Light bulbs in parallel. The first variant again involves a single light bulb. The second variant involves two light bulbs in parallel. Comparisons yield evidence e1' and e2'.

III: Comparison of the brightness of light bulbs in series (I) and in parallel (II). In the first variant, participants compare the brightness of light bulbs in series, and parallel circuits to the one-bulb case only. In the second variant, participants compare series and parallel cases to each other. This produces evidence e $1 "$ and e2".

All six different types of evidence are referred to as an evidence set $\epsilon=\left\{\mathrm{e} 0, \mathrm{e} 1, \mathrm{e} 2, \mathrm{e} 0^{\prime}, \mathrm{e}^{\prime} \mathrm{e} 2^{\prime}, \mathrm{e} 0^{\prime \prime}\right.$, $\left.\mathrm{e}^{\prime \prime}, \mathrm{e} 2^{\prime \prime}\right\}$, with $\mathrm{e} 0, \mathrm{e} 0^{\prime}$ and $\mathrm{e} 0^{\prime \prime}$ representing observations of the brightness of a single light bulb in each context (the brightest light bulb). Further details about the empirical setup, design and excerpts from the student interviews are reported in Koponen et al. (2013); Kokkonen \& Mäntylä (2017), consisting altogether of about 40 cases, from upper secondary level to third year students. When the details are put aside, one finds similar types of five explanatory models from $\mathrm{m}_{1}$ to $\mathrm{m}_{5}$, which can be simplified and idealized as follows:

- $\mathrm{m}_{1}$ : The battery as a source of electricity. Current or voltage may appear as pertinent quantities, synonymously with electricity-.

- m2: Model m1 augmented with idea that components consume electricity. Current or voltage appear as pertinent quantities.

- $\mathrm{m}_{3}$ : Model $\mathrm{m}_{2}$ augmented with idea that voltage/current over components creates/needs current/voltage. Current and voltage appear now to be partially differentiated quantities and related to each other.

- m4: Model m3 refined as scheme based on Ohm's law and Kirchhoff's laws KI and KII. Adequate scientific explanatory model.

- m5: Complete model $\mathrm{m}_{4}$ augmented with the idea that components consume electric energy/power (Joule's law).

The $\mathrm{m}_{1}$ explanatory model is the well-known electric current-based intuitive model found in many empirical studies (see Koponen et al. 2013 and references therein based on a single concept either current or voltage (undifferentiated from each other). This model provides seemingly acceptable explanations in very simple situations (case I). Model $\mathrm{m}_{2}$ operates with two concepts, not totally differentiated, but one of them (current or voltage) is associated with the conservation law D1 (Kirchhoff's law I or II, depending on the case). This model provides acceptable explanations in cases I and II. Model $\mathrm{m}_{3}$ is a partially correct explanatory model operating with two concepts, and where the concepts are differentiated, and where two conservation laws D1 and D2 (Kirchhoff's I and II laws) needed for differentiation are operational. It is understood that voltage creates current (or that voltage can be deduced from a known current). This model provides explanations in cases I and II and also partly for III. Model $\mathrm{m}_{4}$ is the complete and correct scientific model, otherwise similar to $\mathrm{m}_{3}$ but now the relation between the two concepts is explicit (Ohm's law D3). This model provides all the explanations for I-III. Finally, model $\mathrm{m}_{5}$ is as $\mathrm{m}_{4}$ but augmented with notion that in fact the brightness of bulbs, in case of different bulbs, is determined by the electric power they consume (Joule's law D4). A more detailed description of these cases are given in previous studies (Koponen \& Kokkonen 2104; Kokkonen \& Mäntylä 2017) and are not reproduced here.

The structure of the constellation of models (i.e. theory, if one wishes to use the expression) formed by these five explanatory models can be schematically represented as in Figure 1 as a generic tiered system, where more sophisticated models are at the highest tiers and the less 
sophisticated models at lower tiers can be seen as incomplete or partial versions of the higher tier models. Assuming that models $\mathrm{m} 1-\mathrm{m} 5$ revealed by the empirical investigation are actually reflections of the targeted hierarchical levels and models within them, we may use an idealized scheme of stratifications where simpler models are produced (deduced or simply pruned) out of more complex ones. Lower tiers contain partial and incomplete information of possible correct connections but otherwise operate in a similar way. Figure 1 shown in an idealized level of such a tiered system as a descending deductive tree, where two types of basic triads are used recursively, at each level in more incomplete ways.

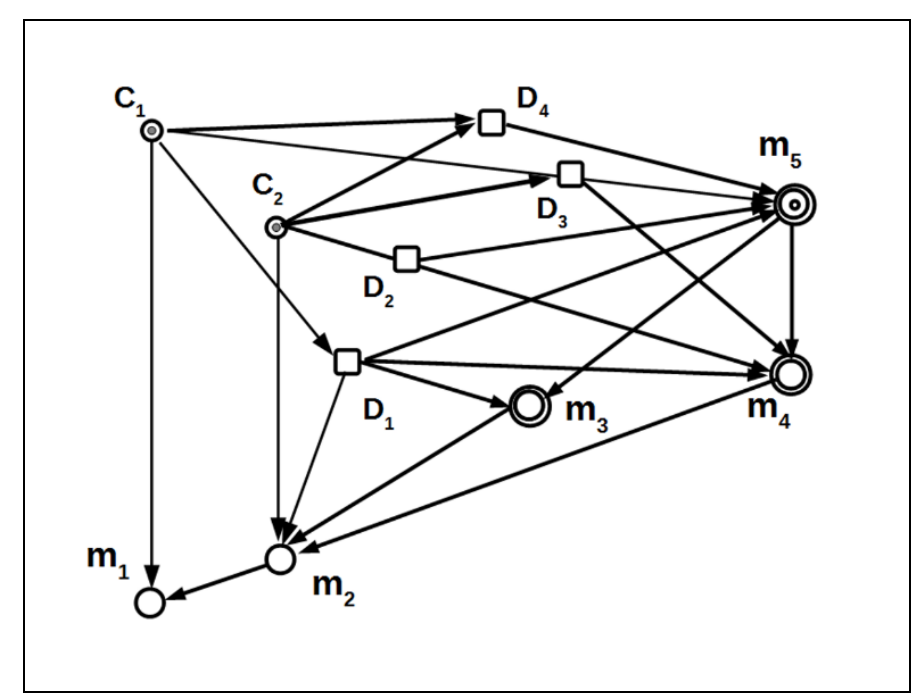

Figure 1. A Tiered system of five explanatory models. The hierarchical levels consist of models $\mathrm{m}_{1}-\mathrm{m}_{5}$ in ascending level of complexity and expanding coverage of explanatory power.

\section{Complex Dynamic Systems View on the Formation of Robust Learning Outcomes}

In the tiered system shown in Figure 1 the model space is hierarchically arranged from the lowestlevel model $\mathrm{m}_{1}$ to the highest-level model $\mathrm{m}_{5}$, so that lower levels are deducible from higher levels, and with each model $\mathrm{m}_{\mathrm{k}}$ getting support from evidence to a certain degree. The structure of the learning task itself is such that it guides the adoption of the models from $m_{1}$ to $m_{5}$, in ascending tiers. The design of the teaching sequence is namely based on the idea of what kind of knowledge and what kinds of explanatory models are expected to be learned at different stages during the learning sequence, and what kinds of competence or proficiency these stages require from a learner. Students' learning outcomes and robust states may not then be actual reflections of the students' internal intuitive conceptions but rather dynamically trapped states, related to the students' dynamic development during the learning stage as it is guided by the task structure.

The design of the learning task and its organization as a teaching sequence can be considered as a kind of pre-defined epistemic landscape, where learning is assumed to take place. The epistemic landscape provides an idealized representation of the target levels of teaching and a description how the target levels are thought to trigger learning in the course of the teaching sequence. The epistemic landscape constitutes of rational utilities of the targeted explanatory models, where models form a hierarchical system. The utility of an explanatory model can be regarded as a balance between the theoretical complexity of the model and evidence that it explains (Koponen \& Kokkonen 2014, Koponen et al. 2016). In simple situations (with little evidence) simple models with limited explanatory power are favoured, but in complex situations 
(with a broader range and possibly structured evidence) complex models with high explanatory power gain utility.

The epistemic landscape is description of the environment that is designed to trigger learning; tasks are produced to facilitate the desired outcomes, which is the target knowledge. The crucial question is, how this design affects the learner's cognitive dynamics and through what kinds of processes. In addition, we draw insight from the description of discovery of new knowledge as it is described as foraging within the epistemic landscape, where the structure of the landscape guides the foraging processs (Weisberg \& Muldoon 2009; McKenzie et al. 2015). In this case, foraging proceeds in the direction of the steepest growth of utility, with increased utility thus encouraging the foraging to continue in the direction of growing utility. When a learner applies a given model successfully, it increases the proficiency (learning has taken place, increasing the learner's future chances for success), but if the explanation requires decreased utility, this reduces the proficiency (Koponen et al. 2016).

\section{Simulation Model of a CDS View of Robust Learning Outcomes}

Here, we focus on a three-tiered system consisting of five models as represented in Figure 1. To each model we attach an utility $u_{k}, k=1 . . .5$ which provides us with an abstract representation of the likelihood of a given explanation. Such a rational utility $u_{k}$ of the model $m_{k}$ depends on two external (exogenous) variables: the evidence $\epsilon$, which is assumed to be cumulative so that $\epsilon>\epsilon^{\prime}$ contains all the evidence contained in $\epsilon^{\prime}$, and on proficiency $\kappa$ which describes the learners' proficiency to use the model in giving an explanation (i.e. competence in using the theory). In what follows, we use the continuous parameters $\epsilon \in[0,1]$ to describe evidence so that $\epsilon=1$ represents the full evidence set $\epsilon=\left\{\mathrm{e} 0, \mathrm{e} 1, \mathrm{e} 2, \mathrm{e} 0^{\prime}, \mathrm{e} 1^{\prime} \mathrm{e} 2^{\prime}, \mathrm{e} 0^{\prime \prime}, \mathrm{e} 1^{\prime \prime}, \mathrm{e} 2^{\prime \prime}\right\}$. The parameter $\kappa \in[0,1]$ describes the proficiency where 1 denotes full mastery of the highest-level model and the ability to derive all the lower level models from it.

In the epistemic landscape the models m1-m5 are thus not directly the explanatory models derived from empirical research. Instead they should be taken as the targeted models that can be deduced or derived through rational arguments from higher level models. In this sense, the epistemic landscape represents different levels of sophistication of the targeted explanatory models. The hierarchy of tiered system of models is reflected also in the construction of the utility functions so that the functions corresponding to the higher models are recursively based on functions describing the lower models. The resulting set of functions are shown in Table 1. Only the generic form of utility functions are needed, and all results are of qualitative rather than of quantitative significance. Here, the utility functions are chosen to be simple MinMax distribution functions because of their convenient mathematical properties (Jones 2009). The utility associated with these kinds of models is then rational in the sense that it depends on the possibility to deduce the lower level models from higher level models. Figure 2 shows a sketch of an epistemic landscape spanned by such rational utilities corresponding to the tiered system of models in Figure 1. The height of the landscape describes the likelihood that the model explains i.e., has high utility. The epistemic landscape is a manifold of five layers, each layer representing the utility of one model and having a peak in the region where the utility is the highest. This region is the targeted knowledge at a given stage of the learning sequence. The higher the utility of a given model, the higher is the likelihood of adopting that model in the space spanned by variables $\kappa$ and $\epsilon$. In the tiered structure of models the models $\mathrm{m}_{1}$ and $\mathrm{m}_{2}$ require only the low proficiency proficiency $(0.0<\kappa<0.33)$, model $\mathrm{m} 3$ require intermediate proficiency $(0.33<\kappa<0.66)$, while models $\mathrm{m} 4$ and $\mathrm{m} 5$ require the highest levels of proficiency $(0.66<\kappa<1.00)$. In ideal case, a student 
Table 1: The utility functions $u(\epsilon, \mathcal{K})$ forming the epistemic landscape. The normalization factors $\mathrm{N}_{1}-\mathrm{N}_{5}$ are defined so that maximum value of each utility function is 1 . The functions describing rational utilities $f_{\mathrm{n} 1, \mathrm{~m} 1}(\epsilon, \mathcal{K})=x^{\mathrm{m}}\left(1-x^{\mathrm{m}-1}\right)^{\mathrm{n}-\mathrm{m}}$ are MinMax-distributions (Jones 2009).

\begin{tabular}{|c|c|}
\hline Model & Utility function \\
\hline $\mathrm{m} 1$ & $u_{1}(\epsilon, \mathcal{K})=N_{1} f_{\mathrm{nn} 1, \mathrm{~m} 1}(\epsilon, \mathcal{K}) f_{\mathrm{n}^{\prime} 1, \mathrm{~m}^{\prime} 1}(\epsilon, \mathcal{K})$ \\
\hline $\mathrm{m}_{2}$ & $u_{2}(\epsilon, \mathcal{K})=N_{2}\left[a_{1} u_{1}(\epsilon, \mathcal{K})+a_{2} f_{\mathrm{n} 2, \mathrm{~m} 2}(\epsilon, \mathcal{K}) f_{\mathrm{n}^{\prime} 2, \mathrm{~m}^{\prime} 2}(\epsilon, \mathcal{K})\right]$ \\
\hline $\mathrm{m}_{3}$ & $u_{3}(\epsilon, \mathcal{K})=N_{3}\left[b_{1} u_{2}(\epsilon, \kappa)+b_{2} f_{\mathrm{n}_{3}, \mathrm{~m} 3}(\epsilon, \mathcal{K}) f_{\mathrm{n}^{\prime} 3, \mathrm{~m}^{\prime} 3}(\epsilon, \mathcal{K})\right]$ \\
\hline $\mathrm{m}_{4}$ & $u_{4}(\epsilon, \mathcal{K})=N_{4}\left[c_{1} u_{3}(\epsilon, \mathcal{K})+\mathcal{c}_{2} f_{\mathrm{nn} 4, \mathrm{~m} 4}(\epsilon, \kappa) f_{\mathrm{n}^{\prime} 4, \mathrm{~m}^{\prime} 4}(\epsilon, \kappa)\right]$ \\
\hline $\mathrm{m}_{5}$ & $u_{5}(\epsilon, \kappa)=N_{5}\left[d_{1} u_{4}(\epsilon, \kappa)+d_{2} f_{\mathrm{n} 5, \mathrm{~m} 5}(\epsilon, \kappa) f_{\mathrm{n}^{\prime} 5, \mathrm{~m}^{\prime} 5}(\epsilon, \kappa)\right]$ \\
\hline
\end{tabular}

progresses through targeted high utility regions and eventually, at the end of the learning sequence, reaches the high proficiency high utility model which explains all the observed events contained in the learning task.

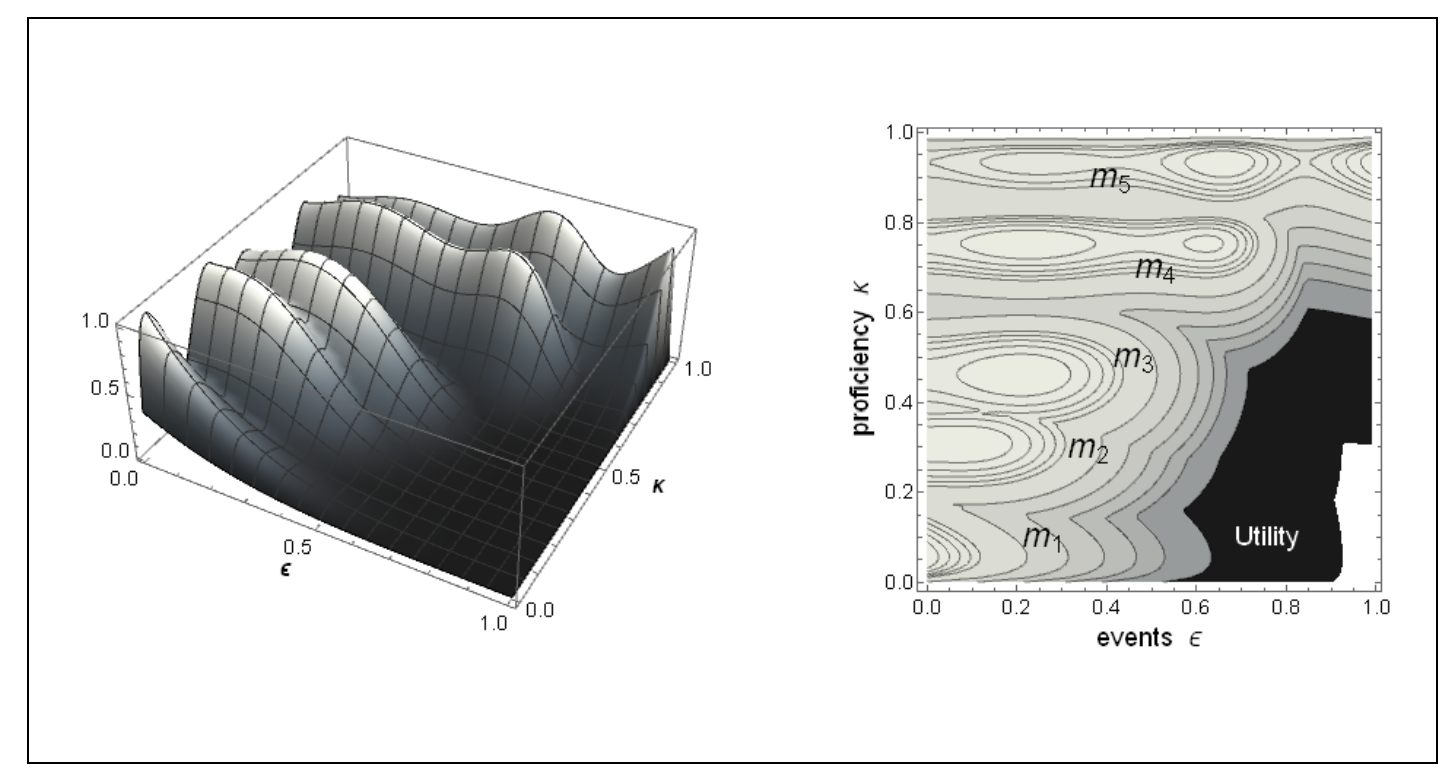

Figure 2. The epistemic landscape consisting of utilities $u_{1}-u_{5}$. The utilities are MinMax-distributions. The parameters are chosen to represent the generic tiered structure of five explanatory models. The diagram on left shows the landscape and the diagram in the right is its representation as a contour plot. The dark regions indicate high and the light regions low the probability. Contours are shown for values $u_{k}=0.9,0.8,0.7,0.6,0.5$, $0.4,0.2$ and 0.1 .

The dynamic evolution of proficiency $\kappa$ has a memory, which depends on the learning history; success increases $\kappa$ while failure to explain decreases $\kappa$. The dynamic evolution of proficiency is assumed to be affected by a memory effect, which depends on the learning history; success increases proficiency while failure to explain decreases it. This can be viewed as a kind of a developmental change which is often described with a logistic model (van Geert 1994; 2014). The resulting logistic model for the development of proficiency $\kappa$ when prior evidence $\epsilon^{\prime}$ is updated to $\epsilon$ is then given by

$$
\kappa(\epsilon) \leftarrow \kappa\left(\epsilon^{\prime}\right)+\delta \mu \kappa\left(\epsilon^{\prime}\right)\left(1-\kappa\left(\epsilon^{\prime}\right)\right), \epsilon^{\prime}<\epsilon \text {, where } \delta= \pm 1 \text { or } 0
$$


where the memory parameter $\mu$ defines the strength of the memory-effect. The increment of proficiency $\kappa$ can be positive $(\delta=1)$, negative $(\delta=-1)$ or zero $(\delta=0)$, which depends on the direction steepest growth/descent of the utility. The memory parameter $\mu$ controlling the strength of the memory can be different for success and failure, but in what follows, for lack of better information, we discuss only the case of equal memory for success and failure.

In the complex dynamic systems (CDS) view on learning the epistemic landscape contains the information of the utilities uk of the k models in a topographical form. The selection of explanatory model is assumed to be fully probabilistic but based on comparison of the rational utility of a given model $\mathrm{k}$ in comparison to utilities of all the other models $\mathrm{j}$. Then the selection of a given model $\mathrm{k}$ from the $\mathrm{N}$ models can be viewed as a probabilistic decision -type problem, in which case the decision to select a given model $\mathrm{k}$ is given as the probability (Yukalov \& Sornette 2014; Koponen et al. 2016)

$$
P_{k}=\frac{u_{k} \exp \left[-\beta u_{k}\right]}{\sum_{j} u_{j} \exp \left[-\beta u_{j}\right]}
$$

where parameter $\beta$ determines the noise-level of selection and is termed in what follows the confidence of choice, in a similar manner as in the probabilistic decision model (Yukalov \& Sornette 2014). Here, the detailed value of $\beta$ is of no consequence provided it is not too small $(\beta<$ $0.5)$ to sweep out the structure of the tiered model space.

In addition to the model selection determined by Eq. (2), another decision to be made is whether or not the chosen model explains the situation adequately. This decision is also made stochastically in each event (i.e. when is increased by $\delta$ ), based on the probability directly proportional on the rational utility. Rational utility, on the other hand, depends on the proficiency, which develops during the learning sequence, guided by the steepest growth/descend of the epistemic landscape consisting of utilities. The success of explanation thus depends on two factors; the model chosen and the rational utility of that model. Consequently, the simulations thus consist of making three probabilistic selections:

1. Probability $P_{k}$ to select a given model $k$ among the all (five) possibilities.

2. Probability $u_{k}$ that model $k$ explains the observation (events).

3. Probability $p_{ \pm}$or $p_{\mathrm{o}}$ that proficiency increases, decreases or remains unchanged.

These outcomes of three possible cases defined above are determined in simulations on a stochastic basis, as an event based Monte Carlo simulation method, where probability to realize the event is proportional to its probability of occurrence. The further details of the technical implementation of probabilistic event based simulations is explained in detail elsewhere (Koponen et al, 2016).

\section{Simulations of Learning and Formation of Attractors}

The outcome of the simulations applied in case of learning the tiered theory structure is number density $\mathrm{nk}$ of choice of given model $\mathrm{mk}$ at given values of and $\kappa$. In addition, we need to consider the utility weighted number density, which is here called pragmatic utility $\Psi \mathrm{k}$ and is roughly given by product uk nk. In both cases attractor type regions are found but the interpretation of these quantities and their relation to the empirical findings is different. The number density nk can be related to the likelihood that a student holds an explanatory model even if it cannot be 
expressed through an explicit explanatory event, while the pragmatic utility $\Psi \mathrm{k}$ is the likelihood that a student expresses (explicates) the model in an event by giving an explanation.

The number density nk is directly related to fraction of students, in an ensemble of students, which at a certain stage are found to hold a given model mk, even in case the model explains little or nearly nothing. Of course, in this case the traditional empirical methods based on interviews or similar kinds of research settings are not likely to reveal that the student after all holds a certain model; there is thus important distinction between holding a model and explicating a model in a given context.

The dynamics changes in holding or selecting more advanced models during the learning (or training sequence) when increases from $=0$ (no evidence) to $=1$ (all evidence available) is clear when the density $\mathrm{nk}(, \kappa)$ of selected models in the $(, \kappa)$-space is examined. The density distribution $\mathrm{nk}(, \kappa)$ of preferred models are shown in Figure 3 for strong $(\mu=0.05)$, intermediate $(\mu=0.04$ and 0.02$)$, and weak $(\mu=0.01)$ memory effects. The results are shown only for cases that initially have proficiencies $0.45<\kappa<0.55$, which represents a middle cohort of initial proficiencies and thus represents the assumed average student for which the learning task is designed. The

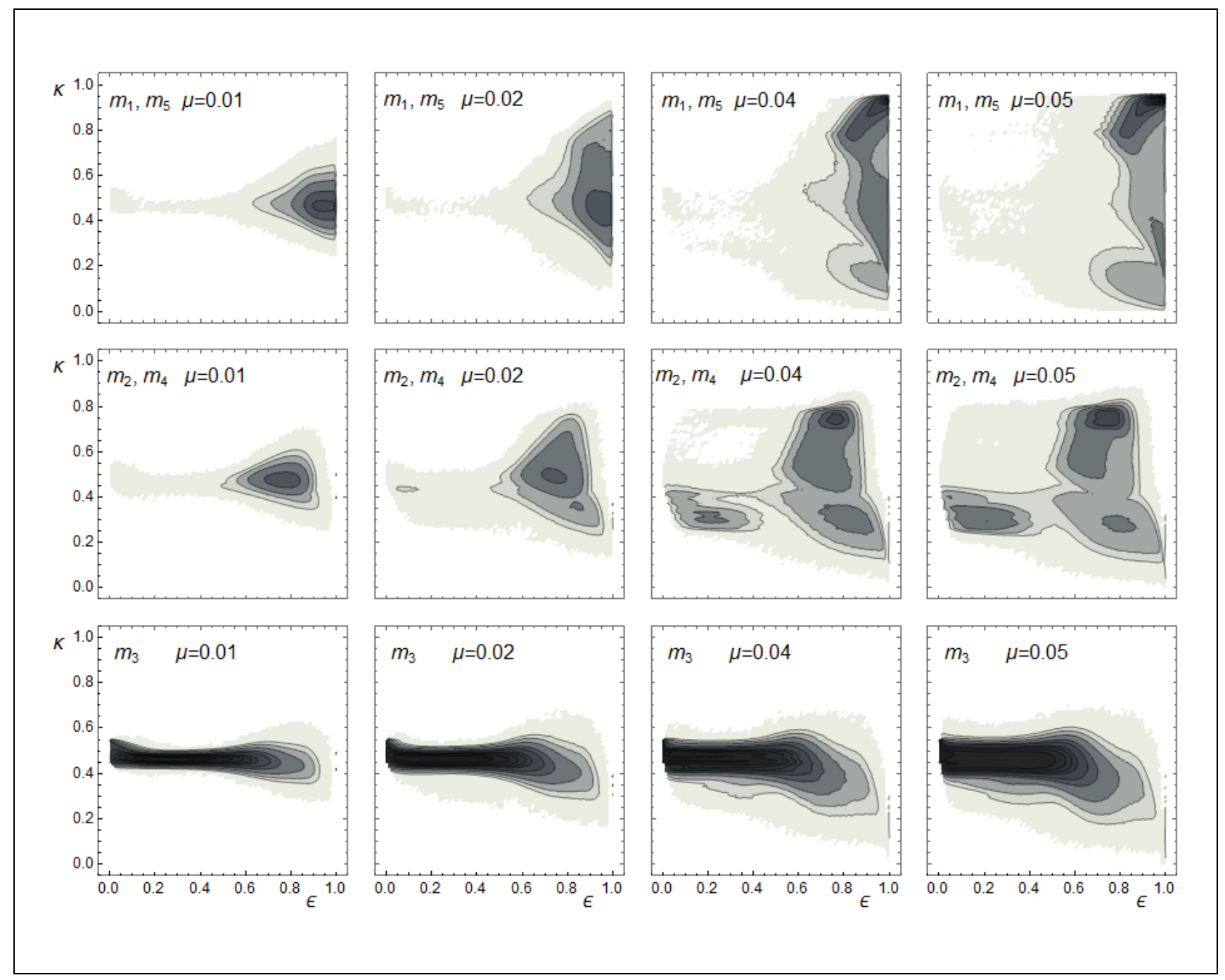

Figure 3. The density distribution $n_{k}(\epsilon, \kappa)$ of preferred models for the density distribution $n_{k}(\epsilon, \mathcal{K})$ of model preference for models $\mathrm{m}_{1}-\mathrm{m}_{5}$ for strong $(\mu=0.05)$, intermediate $(\mu=0.04$ and 0.02$)$, and weak $(\mu=0.01)$ memory effects. Robust, attractor-type states emerge (dark regions) from the accumulation of learning paths. Contours are shown for values $-\ln n_{k}=0.0,0.2,0.4, \ldots$ and so on as steps of 0.2. 
results shown in Fig. 3 demonstrate how selection of given model $\mathrm{k}$ accumulate to certain attractor regions, different from but close to those regions where rational utilities have peak values. This behaviour is due to the dynamic effects of foraging for the best explaining models in the epistemic landscape and how memory affects the development of proficiency.

The density distribution shows directly how likely a selection of given model is in comparison to other models. The attractor-type regions can be discerned as dark areas in the density plot, where the values are normalized so that for the maximum likelihood of most likely model Max[nk]=1 always. When the memory is weak $(\mu \approx 0.01)$ the intermediate level model m3 preferred in middle cohort already in the beginning of the learning sequence, remains preferred throughout the learning sequence. The highest level models $\mathrm{m} 4$ and $\mathrm{m} 5$ remain marginally represented until the very end of the learning sequence. In this case there is thus little if any advancement in learning. When memory increases from $\mu=0.01$ to 0.04 the higher-level models $\mathrm{m} 4$ and $\mathrm{m} 5$ are more likely to be selected. Note, however, that because the memory of failure and success is symmetrical, also selection of lower level models is enhanced, i.e. learning outcomes begin to be polarized because memory affects the learning history. Of course, for completely asymmetrical memory where only success affects the learning history no polarization would take place. We have here chosen to study only the case of symmetrical memory, which probably is closer to the real situation where students after all are negatively affected by experiences of failure. For the highest memory $\mu=0.05$ the development is very regular, models $\mathrm{m} 1, \mathrm{~m} 2$ are likely choices only at low proficiencies at the beginning of the training sequence for $<0.3$, the model $\mathrm{m} 3$ and $\mathrm{m} 4$ in the middle of the training sequence $0.6<\quad<0.3$ and finally, at the end of the training sequence $>0.6$ the model $\mathrm{m} 5$ is dominant.

The distributions $\mathrm{nk}(, \kappa)$ of model preference in all cases show clear attractor-type regions in $(, \kappa)$-space; sharper ridges or islands of preferred models are formed. For high memory-effects and high confidence the attractor-type regions are sharply defined, island-like and give expression of well-focused models with no overlap with any other explanatory model. The overall picture is then that when new evidence becomes available, the learner switches to better explaining models and lower level models disappear towards the end of the learning sequence. This is the successful learning path. Accumulation of densities nk in certain regions are those areas, where empirical research will most likely find a certain conception if it looks for how certainly a belief is held but not so much how the model is explicated. If this interpretation is correct, the vision of conceptual change as a switch between cognitively pre-existing static states to another needs to be questioned and replaced by a more dynamic and fluid picture of dynamically formed robust attractor type states.

The likelihood of expressing a model is related to nk but in addition to that the model must also provide an explanation; it should have a high enough utility. Obviously, if the preferred model explains little, the empirical situation does not provide possibilities for a student to explicate the model. In such an empirical situation only those models the students hold and which they explain become visible. The information of explicated models is carried by the pragmatic utility $\Psi \mathrm{k}$ as defined by selection rules (1) and (2) taken together. In practice pragmatic utility is roughly the density nk weighted with corresponding rational utility function i.e., $\Psi \mathrm{k} \sim \mathrm{uk} n \mathrm{k}$. In most (but not all) empirically investigated cases the pragmatic utility seems to be more relevant for comparisons with empirical results. In some versions of "clinical interviews" (diSessa 1993; diDessa et al. 2004; Russ et al 2012), however, which explore the students' understanding more indirectly with in-depth questioning it might be possible that $\mathrm{nk}$ rather than $\Psi \mathrm{k}$ is obtained. The pragmatic utility takes into account not only the selection of model but also whether or not the 
model explains the phenomenon under investigation. Figure 4 shows the pragmatic utility corresponding to the densities shown in Fig. 3.

Because pragmatic utilities have similarly low overlaps as the rational utilities, we can display several of them in the same panel. For comparison, the effect of initial cohort of proficiencies on the formation of the attractor-states is shown in lower panel in Fig. 4. When the initial cohort consists of low-proficiency students (high-proficiency cohorts are uninteresting since they remain in the high proficiency region) the learning is more polarized because low-proficiency students are more likely affected by negative feedback (failure in explaining) than more proficient students. In short, this shows how success and failure both are easily self-reinforcing drivers of learning.
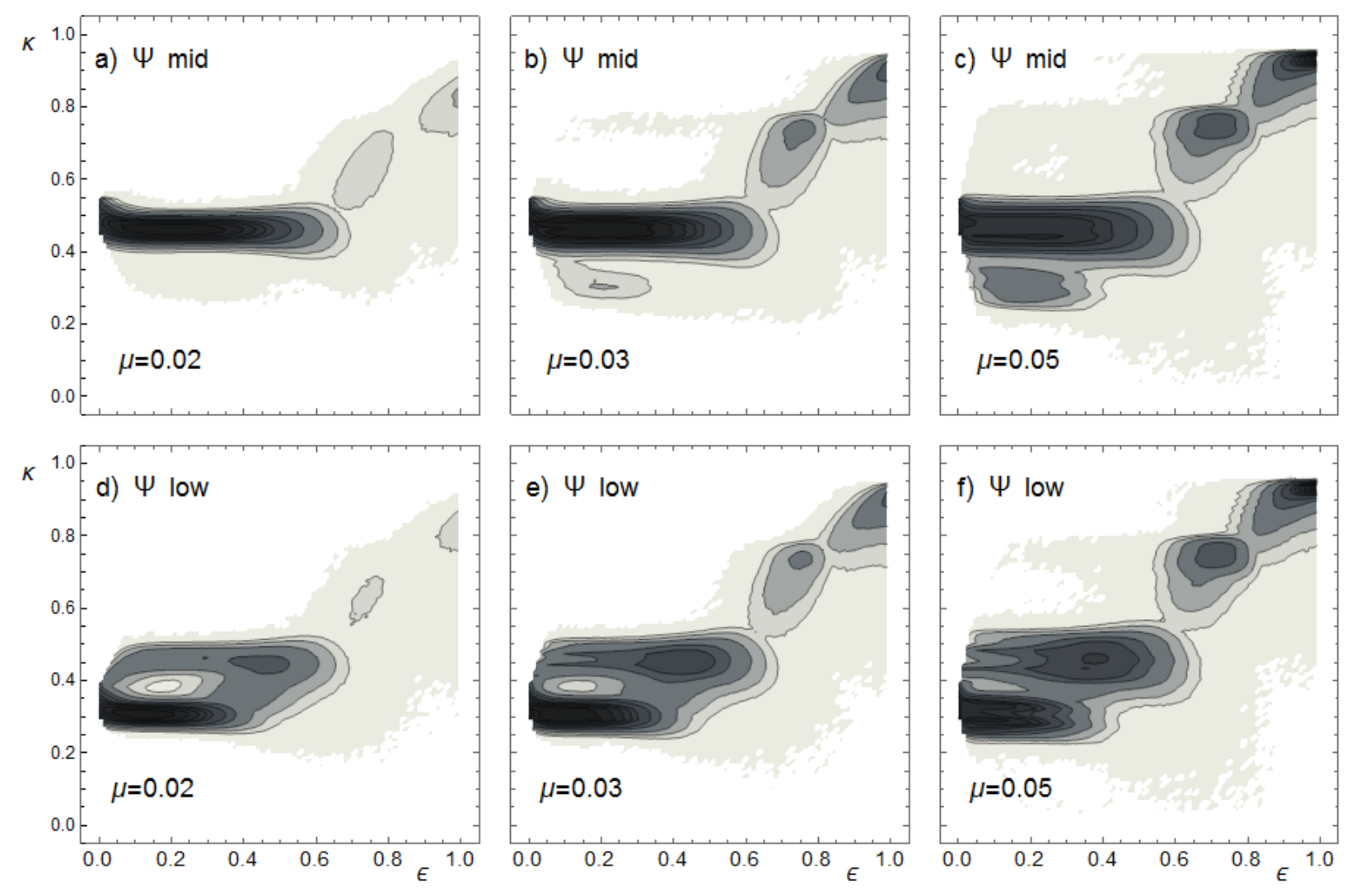

Figure 4. The pragmatic utility $\Psi_{k}(\epsilon, \kappa)$ of models, $\mathrm{m} 1-\mathrm{m} 5$ for $\mathrm{m} 5$ for different memories $\mu=0.02,0.03$ and 0.05 . The confidence is in all cases is $\beta=5.0$. For each set, $\operatorname{Max}\left[\Psi_{1}(\epsilon, \kappa), \ldots, \Psi_{5}(\epsilon, \kappa)\right]=1$ and contours are shown for the values $-\ln \Psi_{k}=0.0,0.2,0.4, \ldots$ and so on with steps of 0.2 . The upper panel shows results for the initial mid-cohort $(0.45<k<0.55)$ and the lower panel for the low-cohort $(0.30<k<0.40)$.

\section{Discussion and Conclusions}

In the CDS view, the formation of robust learning outcomes accrues from foraging in an epistemic landscape, which represent the target knowledge as it is contained in the designed learning task. The interplay of students' cognitive dynamics and the target knowledge as it appears in the design of the learning tasks leads to the formation of attractor-type regions of preferred explanatory models in an epistemic landscape. The origin of these robust states is in the learning dynamics and 
how it interacts with the context (structure of the learning task). In some cases, depending on the learner's proficiency and the development of the proficiency, learning outcomes may match the target knowledge, but in some other cases, they may fall short of the targeted outcomes. However, even those states not matching the targeted states are robust, thus giving the impression of preexisting conceptual states of learners as is assumed in traditional conceptual change models.

In the CDS-view the picture of conceptual change as a switch from intuitive conceptions to more scientific conceptions (or sometimes, to other intuitive conception) emerges as a rapid dynamic change from one attractor to another of the state rather than a switch from one static state to another. The fact that for most of the training sequence there is little overlap between the different attractors and thus little chances for clearly continuous change, gives an obvious picture of discontinuous switching from robust state to another. This course of events corresponds the traditional view of conceptual change. However, the CDS-view strongly suggests that behind this behaviour is after all continuous learning dynamics and which, through rational utilities, is essentially context dependent. In regard to conceptual change research the CDS-view suggests we pay attention to the difference between holding a model (visible in the density of the selected states nk) and expressing a model (visible as pragmatic utility $\Psi \mathrm{k}$ ) is relevant for the interpretation of the empirical findings. This difference may well be present also in different empirical research settings. Some research settings based on the so called "clinical" interviews (diSessa 1993; diDessa et al. 2004; Russ et al 2012) allow rather spontaneous explication of explanations, in which case one may assume that even if a learner knows that a certain model does not explain a phenomenon well, the learner is able to give other indications of the preferred model during the interview. In this case, the density $\mathrm{nk}$ of selected model is probably closer to the empirically available information than the pragmatic utility $\Psi \mathrm{k}$. If one assumes that $\mathrm{nk}$ is accessible in clinical interviews, this is in agreement with finding that the outcome of clinical interviews is a dynamically coexisting explanatory models (diSessa 1993; Clark 2006; Hammer et al. 2005) instead a set of more coherent and sharply defined explanatory models. On the other hand, in some research settings when interviews are structured and explanations are required, or students are working with tutorial-type tasks, one may expect that information about explanatory models becomes accessible only if the learners can really produce explanations, that is, explicate their explanatory models in the given context (McDermott, 1992; Engelhardt \& Beichner 2004; Koumaras et al. 1997; Koponen \& Kokkonen 2014). In these cases we may assume that the accessible states are those corresponding to the pragmatic utility $\Psi \mathrm{k}$, rather than the density nk. In this case the structure of the set of attractors corresponds more closely to the structure of the epistemic landscape; the overall picture is then of more coherent explanatory models with little overlap. This difference between the selection of models and explicated explanations, reflected in the difference between attractors corresponding to $\mathrm{nk}$ and $\Psi \mathrm{k}$ may be one possible reason behind the different interpretations of interview outcomes in seemingly similar situations.

In summary, the dynamic view provides a fresh viewpoint on conceptual change and suggests new ways to conceptualise it. The results we have provided here are far from conclusive and are at best only suggestive, but we think that the view proposed here of learning outcomes as context dependent, dynamically robust but ultimately an emergent epiphenomena deserves closer attention and has prompted us to design very different empirical research settings. If correct, it may resolve many of the dead-ends of the traditional conceptual change research and point to in new directions for the design of learning tasks and settings. 


\section{References}

Brown, D. E. (2014). Students' Conceptions as Dynamically Emergent Structures. Science \& Education, 23, 14631483.

Brown, D. E., \& Hammer, D. (2008). Conceptual change in physics. In S. Vosniadou (Ed.), International handbook of research on conceptual change (pp. 127-154). New York, NY: Routledge.

Chi, M. T. H. (2005). Commonsense Conceptions of Emergent Processes: Why Some Misconceptions Are Robust. The Journal of the Learning Sciences, 14, 161-199.

Chi, M. T. H., \& Slotta, J. D. (1993). The Ontological Coherence of Intuitive Physics. Cognition and Instruction, 10, 249-260.

diSessa, A. A. (1993). Toward an Epistemology of Physics. Cognition and Instruction, 10, 105-225.

diSessa, A., Gillespie, N. M., Esterly, J. B. (2004) Coherence versus fragmentation in the development of the concept of force. Cognitive Science, 28, 843-900.

diSessa, A. A., \& Sherin, B. L. (1998). What Changes in Conceptual Change? International Journal of Science Education, 20, 1155-1191.

Engelhardt, P. V. \& Beichner, R. J. (2004). Students' Understanding of Direct Current Resistive Electrical Circuits. American Journal of Physics, 72, 98-115.

Gupta, A., Hammer, D. \& Redish, E. F. (2010). The Case for Dynamic Models of Learners' Ontologies in Physics. The Journal of the Learning Sciences, 19, 285-321.

Gupta, A.G. \& Wong, A.F. (1985). On Three and Five Parameter Bivariate Beta Distributions. Metrika 32, 85-91.

Hammer, D., Elby, A., Scherr, R. E. \& Redish, E. F. (2005) Resources, Framing, and Transfer. In: Mestre, J. (ed) Transfer of Learning from a Modern Multidisciplinary Perspective (pp 89-119) Greenwich, CT: information Age.

Hammer, D., Gupta, A., \& Redish, E. F. (2011). On Static and Dynamic Intuitive Ontologies. The Journal of the Learning Sciences, 20, 163-168.

Jones, M. C. (2009). Kumaraswamy's Distribution: A beta-type Distribution with Some Tractability Advantages. Statistical Methodology, 6, 70-81.

Kokkonen, T. \& Mäntylä, T. (2017). Changes in university students' explanation models of dc circuits. Research in Science Education, in print.

Koponen, I. T. \& Kokkonen T. (2014). A Systemic view of the learning and differentiation of scientific concepts: The case of electric current and voltage revisited. Frontline Learning Research 4, 140-166.

Koponen, I. T., Kokkonen T. P. \& Nousiainen, M. K. (2016). Dynamic systems view of learning a three-tiered theory in physics: robust learning outcomes as attractors. Complexity, 21, 259-267.

Koumaras, P., Kariotoglou, P., \& Psillos, D. (1997). Causal Structures and Counter-intuitive Experiments in Electricity. International Journal of Science Education, 19, 617-630.

Lee, Y. \& Law, N. (2001). Explorations in Promoting Conceptual Change in Electrical Concepts via Ontological Category Shift. International Journal of Science Education, 23, 111- 149.

McDermott, L. C., \& Shaffer, P. S. (1992). Research as a Guide for Curriculum Development: An Example from Introductory Electricity. Part I: Investigation of Student Understanding. American Journal of Physics, 60, 9941003.

McKenzie Alexander, J; Himmelreich, J. \& Thompson, C. (2015). Epistemic Landscapes, Optimal Search and the Division of Cognitive Labor. Philosophy of Science, 76, 225-252.

Ozdemir, G., \& Clark, D. B. (2007). An Overview of Conceptual Change Theories. Eurasia Journal of Mathematics, Science \& Technology Education, 3, 351-361.

Reiner, M., Slotta, J. D., Chi, M. T. H., \& Resnick, L. B. (2000). Naive Physics Reasoning: A Commitment to Substance-Based Conceptions. Cognition and Instruction, 18, 1-35.

Russ, R. S., Lee, V. R. \& Sherin, B. L. (2012) Framing in Cognitive Clinical Interviews About Intuitive Science Knowledge: Dynamic Student Understandings of the Discourse Interaction. Science Education, 96, 573-599.

Slotta, J. D. \& Chi, M. T. (2006). Helping Students Understand Challenging Topics in Science Through Ontology Training. Cognition and Instruction 24, 261-289.

van Geert, P. (1994). Dynamic Systems Model of Cognitive Growth. In: A Dynamic Systems Approach to Development, Eds. Smith, L., Thelen, E. (pp 265-331) MIT Press, Massachusetss MA.

van Geert, P. (2014). Dynamic Modeling for Development and Education: From Concepts to Numbers. Mind, Brain and Education, 8, 57-73. 
Vosniadou, S., \& Skopeliti, I. (2014). Conceptual Change from the Framework Theory Side of the Fence. Science \& Education, 23, 1427-1445.

Yukalov, V.I. \& Sornette, D. (2014). Self-organization in Complex Systems as Decision Making. Advances in Complex Systems, 17, 1450016.

Weisberg, M. \& Muldoon, R. (2009). Epistemic Landscapes and the Division of Cognitive Labor. Philosophy of Science, 76, 225-252.

Wong, C. L., Chu, H.-E. \& Yap, K. H. (2016) Are Alternative Conceptions Dependent on Researchers' Methodology and Definition?: A Review of Empirical Studies Related to Concepts of Heat. International Journal of Science and Mathematics Education 14, 499-526.

\section{About the Authors}

Ismo T. Koponen is Professor of physics in University of Helsinki, Department of Physics, Finland. He heads the group of didactic physics, and regularly teaches courses on conceptual structure of physics and physics history. His research focuses on cognitive aspect of concept learning and conceptual systems, from viewpoint of complex systems and complex network approaches. Correspondence: ismo.koponen@helsinki.fi

Tommi Kokkonen is a doctoral student of didactic physics in University of Helsinki, Department of Physics, Finland. His research focused in didactic physics, from viewpoint of relational theory of knowledge and systemic view on knowledge.

Maija Nousiainen is Adjunct Professor of didactic physics in University of Helsinki, Department of Physics, Finland. Her research and teaching are connected to physics teacher education and didactic physics. She regularly teaches didactic physics courses for pre-service teachers. Her current research focuses on critical thinking, argumentation and uses of concept networks in knowledge organization.

(c) Copyright 2017. The authors, ISMO T. KOPONEN, TOMMI KOKKONEN AND MAIJI NOUSIAINEN, assign to the University of Alberta and other educational and non-profit institutions a non-exclusive license to use this document for personal use and in courses of instruction provided that the article is used in full and this copyright statement is reproduced. The authors also grant a non-exclusive license to the University of Alberta to publish this document in full on the World Wide Web, and for the document to be published on mirrors on the World Wide Web. Any other usage is prohibited without the express permission of the authors. 